\title{
RESEARCH HIGHLIGHT ALPK1: innate attraction to the sweetness of bacteria
}

\author{
Yansong Xue ${ }^{1}$ and Si Ming Man (D) \\ Cell Research (2018) 28:1125-1126; https://doi.org/10.1038/s41422-018-0100-0
}

\begin{abstract}
Pattern-recognition receptors (PRRs) have critical roles in mediating the recognition of microbial and danger signals. A recent study by Zhou et al. published in Nature demonstrates that ALPK1 is a cytosolic PRR, which recognizes the bacterial metabolite ADP-Hep, triggering activation of the inflammatory NF-KB signaling pathway.
\end{abstract}

Toll-like receptors (TLRs), Nucleotide-binding oligomerization domain (NOD)-like receptors (NLRs), retinoid acid-inducible gene I (RIG-I)-like receptors (RLRs), C-type Lectin receptors (CLRs), AIM2like receptors (ALRs) and the related DNA and RNA sensors are key families of pattern-recognition receptors (PRRs). Pathogenassociated molecular patterns (PAMPs) are recognized by PRRs and trigger an intracellular signaling cascade leading to inflammatory responses and anti-microbial activities. LPS, an essential structural component of the outer membrane of Gram-negative bacteria, is a strong activator of the NF-KB and inflammasome pathways. Recent studies have shown that the LPS metabolite Dglycero- $\beta$-D-manno-heptose 1,7-bisphosphate (HBP), can also activate the innate immune system, via a poorly characterized pathway involving the kinase ALPK1 (also known as Alpha-protein kinase 1 or Lymphocyte alpha-protein kinase) and the adaptor protein TIFA (also known as TRAF-interacting protein with FHA domain-containing protein A). ${ }^{1-5}$

In a new study published in Nature, Zhou and colleagues performed a transposon mutagenesis study of 21,000 mutants of the Gram-negative bacterium Yersinia pseudotuberculosis and found that a bacterial transposon carrying a hldE mutation had an impaired ability to induce activation of NF-KB in the human embryonic kidney cell line $293 T .{ }^{6}$ The gene $h l d E$ is essential for the biosynthesis of ADPHep. Moreover, deletion of related genes required for the biosynthesis of the ADP-Hep-related metabolite, HBP, prevented activation of NF-KB by $Y$. pseudotuberculosis. In addition to $Y$. pseudotuberculosis, the Gram-negative bacteria Escherichia coli (DAEC), enterotoxigenic E. coli (ETEC) and Burkholderia cenocepacia required HIdE for the activation of NF-KB. Further, purified ADP-Hep can induce NF-KB activation and IL-8 secretion in 293T cells, demonstrating that ADP-Hep is a bona fide PAMP (Fig. 1).

To identify the innate immune sensor recognizing ADP-Hep, Zhou and colleagues used a FACS-based, genome-wide CRISPRCas9 screen on a 293T NF-KB reporter cell line. They found that deletion of genes encoding ALPK1, TIFA and TRAF6 impaired ADPHep-induced NF-KB activation and IL-8 expression in 293T cells (Fig. 1). These results highlight that ALPK1 and TIFA are essential mediators of NF-KB activation in response to ADP-Hep. This finding is supported by previous studies showing that HBP, found in the bacteria Neisseria meningitidis, Shigella flexneri, Salmonella enterica serovar Typhimurium and Helicobacter pylori, induce activation of ALPK1-TIFA-dependent NF-KB signaling in host cells ${ }^{1-5}$ (Fig. 1). Importantly, Zhou and colleagues noted that HBP failed to directly activate ALPK1; instead, HBP is converted by host-derived adenylyltransferases to ADP-heptose 7-P, a substrate which can then activate ALPK1 (Fig. 1).

Zhou and colleagues proceeded to elucidate the crystal structure of the N-terminal domain of ALPK1 and showed that ADP-Hep specifically binds to the pocket-like structure of ALPK1. Introducing several mutations at the $\mathrm{N}$-terminal domain of ALPK1 impaired the ability of ALPK1 to activate TIFA in response to ADP-Hep. Both the Nterminal domain and the kinase domain of ALPK1 were essential for the functional activity of ALPK1, and ALPK1, TIFA and TRAF6 coimmunoprecipitated in the presence of ADP-Hep. Zhou and colleagues further demonstrated a physiological effect of ALPK1 in a mouse model. They found that wild-type mice injected with either ADP-Hep or B. cenocepacia generated a robust NF-KB-dependent inflammatory response, whereas $A l p k 1^{-1-}$ mice failed to do so. ${ }^{6}$

The collective studies by Zhou et al. and others identified a new cytosolic PRR specialized in the recognition of microbial cell wall components $^{1-6}$ (Fig. 1). The ALPK1-TIFA pathway is analogous to the cytosolic NOD1 and NOD2 pathways driving activation of NF$K B$ in response to peptidoglycans. These findings broaden our knowledge of the expanding innate immune surveillance system and further illustrates immunological redundancies in the recognition of microbial components. Importantly, the availability of a cytosolic sensor that recognizes intermediate molecules of the LPS biosynthesis pathway suggests that the host can detect bacteria even if they were to alter their LPS structure to evade the LPS-sensing receptors TLR4 and caspase- 11 .

Several questions remain to be explored. How is ALPK1 able to discriminate commensal bacteria and pathogens given that both can synthesize ADP-Hep? Zhou et al. have shown that the type III secretion system of $Y$. pseudotuberculosis is required for activation of NF-KB signaling, indicating that a bacterial injection system mediated ADP-Hep translocation to induce activation of ALPK1 (Fig. 1). This mode of ligand delivery allows the host cell to recognize ADP-Hep from pathogens encoding a type III secretion system. However, extracellular ADP-Hep can also freely penetrate the host cell membrane and access the host cytoplasm, and bacteria that do not encode type III secretion systems can also activate ALPK $1^{6}$ (Fig. 1). In this case, how might host cells avoid responses to ADPHep produced by commensal bacteria?

Recognition of HBP by ALPK1 appears to be more regulated. HBP requires translocation to the host cytoplasm by the type IV secretion system. ${ }^{4,5,7}$ In addition, HBP must be converted by host adenylyltransferases to ADP-heptose 7-P in order to activate ALPK $1,{ }^{6}$ ensuring an extra layer of stringency prior to activating the immune system (Fig. 1). Therefore, recognition of HBP appears to be more selective towards pathogens; investigation into how pathogens might evade this recognition system would be of interest in future studies. 


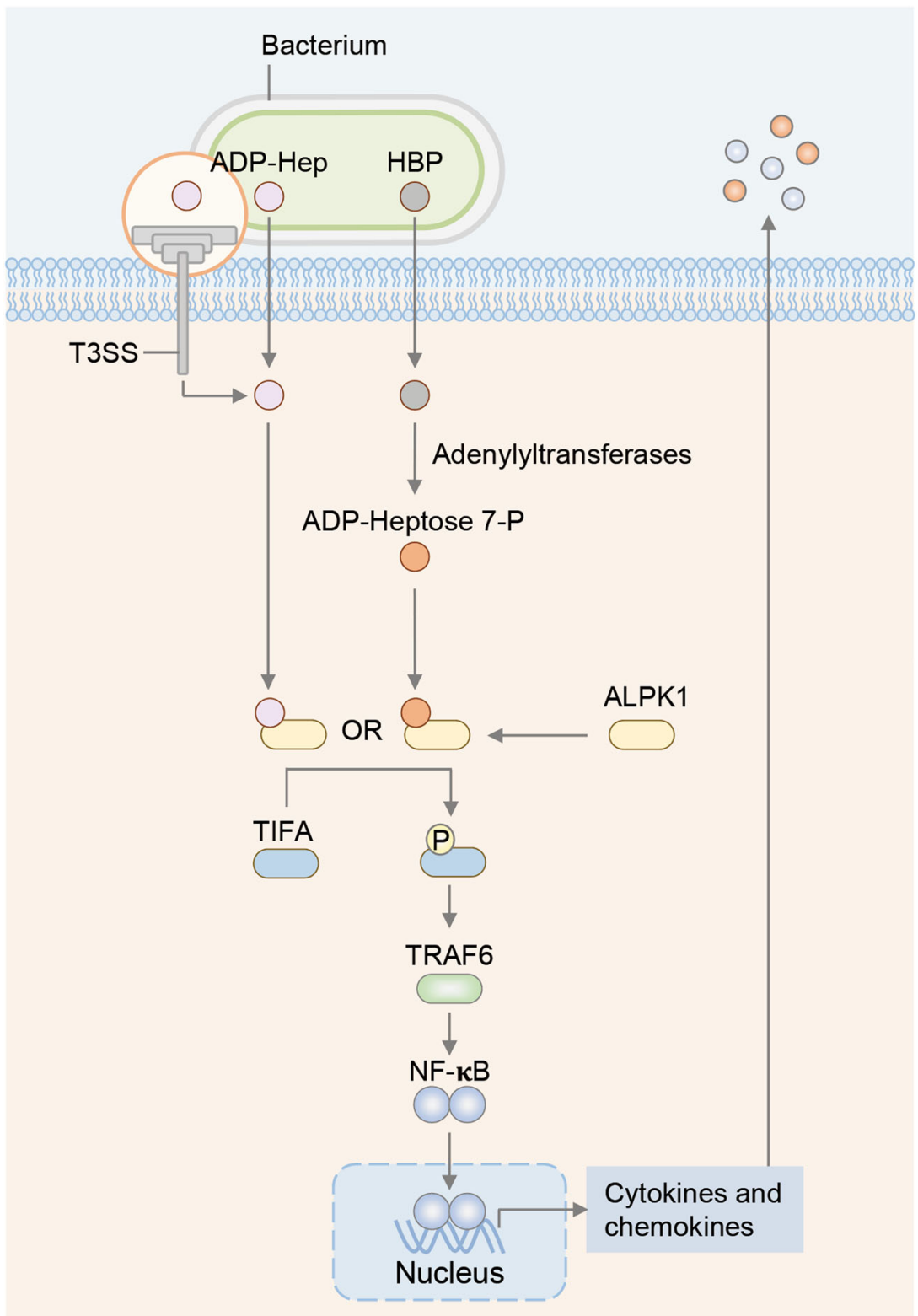

Fig. 1 ALPK1 is a new cytosolic sensor of bacterial ADP-Hep and related sugar molecules

The ALPK1-TIFA signaling pathway has so far been defined mainly in human embryonic kidney cells, intestinal epithelial cells, gastric cells and cervical cancer cells. ${ }^{1-7}$ Could ALPK1 be a major PRR operating in non-immune cells and cells lacking other cytosolic innate immune sensors such as NLRs and ALRs? Together with other studies, the findings by Zhou et al. highlight that ADPHep and HBP are an important class of PAMPs which engages the newly recognized innate immune sensor ALPK1. These findings would enable exploration of novel small-molecule inhibitors or activators for the treatment of pathologies associated with dysregulation of the ALPK1-TIFA signaling pathway, including those seen in gout, chronic kidney disease and cancer. ${ }^{8-11}$

\section{REFERENCES}

1. Gaudet, R. G. et al. Science 348, 1251-1255 (2015).

2. Gaudet, R. G. et al. Cell Rep. 19, 1418-1430 (2017).

3. Milivojevic, M. et al. PLoS Pathog. 13, e1006224 (2017).

4. Stein, S. C. et al. PLoS Pathog. 13, e1006514 (2017).

5. Zimmermann, S. et al. Cell Rep. 20, 2384-2395 (2017).

6. Zhou, P. et al. Nature 561, 122-126 (2018).

7. Gall, A., Gaudet, R. G., Gray-Owen, S. D. \& Salama, N. R. mBio 8, e01168-17 (2017).

8. Wang, S. J. et al. J. Mol. Med. 89, 1241-1251 (2011)

9. Ko, A. M. et al. Int. J. Epidemiol. 42, 466-474 (2013).

10. Yamada, Y. et al. J. Med. Genet. 50, 410-418 (2013).

11. Liao, H. F. et al. Sci. Rep. 6, 27350 (2016). 